\title{
PARP inhibitors in the treatment of ovarian cancer
}

\author{
Alain G. Zeimet · Verena Wieser · Katharina Knoll · Daniel Reimer · Christian Marth
}

Received: 19 December 2019 / Accepted: 12 February 2020 / Published online: 13 March 2020

(c) The Author(s) 2020

\begin{abstract}
Summary The recent exciting findings on the use of poly(adenosine diphosphate [ADP]-ribose) polymerase inhibitor (PARPi) maintenance therapy in the first-line and later lines of treatment in ovarian cancer are illustrated. Unprecedented advantages have been shown in first-line therapy not only in BRCA-mutated cancers, but also in tumor exhibiting a homologous recombination repair deficiency (HRD) unrelated to BRCA aberrations. The advantages of PARP maintenance therapy in around 50\% of HR-proficient highgrade ovarian cancers are far less clear and, even though of statistical significance, the clinical benefit for the patients may be of borderline significance. The pre-treatment testing of HRD remains a matter of debate especially in the light of the current era of precision medicine. Data on the combination of PARPi with bevacizumab maintenance therapy uncovered additive beneficial therapeutic effects. In recurrent ovarian cancer, results on PARPi maintenance therapy after response to platinum-based reinduction chemotherapy are also excellent. At present, however, PARPi maintenance therapy remains reserved for PARPi-naive patients, since the data on PARPi after PARPi is extremely sparse.
\end{abstract}

Keywords Ovarian cancer - PARP inhibitors · Maintenance therapy $\cdot$ HRD $\cdot$ BRCA mutation

\section{Introduction}

The enzyme poly(adenosine diphosphate [ADP]-ribose) polymerase (PARP), was first described by Pierre Chambon in 1963 and was found to be crucially im-

A. G. Zeimet $(\bowtie) \cdot$ V. Wieser $\cdot$ K. Knoll $\cdot$ D. Reimer $\cdot$ C. Marth Department of Obstetrics and Gynaecology, Innsbruck Medical University, Anichstraße 35, 6020 Innsbruck, Austria alain.zeimet@i-med.ac.at plicated in the "base excision repair" of single strand breaks in the DNA [1]. Abrogating the activity of PARP by specific inhibiting drugs leads to an accumulation of unrepaired single strand breaks and consequently to an increased number of double strand breaks (DSB), which in turn are commonly addressed by high-fidelity, template-dependent homologous recombination repair (HRR). Deficiency in the latter repair system, referred to as HRD, is mostly due to a mutation in one of the various genes encoding for proteins involved in the DNA damage repair process, such as BRCA1 and 2, Rad51C, Check 2, Fanconi anemia proteins, and a number of others. Thus, inhibition of PARP in cells with an HRD background leads in particular to cell death due to the accumulation of unrepaired DSBs [2]. This phenomenon characterized by the lack of function of two separate DNA repair systems, the one by inhibitory drugs and the other due to genetic or epigenetic alterations, is referred to as "synthetic lethality." Hence, cancers bearing HRD exhibit the highest susceptibility to respond to PARP inhibitors (PARPi). In this regard, high-grade serous ovarian cancer, which is the most common histological ovarian cancer subtype, represents an ideal candidate for PARPi treatment, since around $50 \%$ of these cancers exhibit the HRD phenotype, of which $20-25 \%$ account for BRCA1 and -2 germline or somatic aberrations [3]. Similar sensitivity for PARPi therapy is also assumed for high-grade endometrioid ovarian cancers [4]. Furthermore, PARPi also impair the non-homologous end-joining (NHEJ) DNA repair mechanism, which act in HRD cells as an alternative rescue DNA repair pathway for DSBs [2]. Usually in ovarian cancer, the HRD phenotype is associated with a better response to platinum-based chemotherapy and thus with a better clinical outcome [6]. Preclinical and early clinical investigations uncovered a strong relationship between the responsiveness to platinum 
and the therapeutic efficacy of PARPi. Consequently, in most clinical studies as well as in routine practice, the response to platinum-based chemotherapy is considered an extremely reliable "functional in vivo biomarker" to predict PARPi responsiveness.

\section{PARPi in recurrent ovarian cancer}

A number of large phase 2 and phase 3 placebocontrolled clinical trials have been published on recurrent platinum-sensitive high-grade ovarian cancer (HGOC), in which so far three different PARPi (olaparib, niraparib, and rucaparib) were tested as daily maintenance therapy until progression in patients that showed response to the actual platinum-based chemotherapy [4, 6-8]. The addition of maintenance therapy with PARPi resulted in an impressive improvement in progression-free survival (PFS) with hazard ratios (HR) from around 0.35 compared to the placebo arms in the intention-to-treat (ITT) populations. As a general rule, efficacy dropped consistently from patients with tumors mutated in the BRCA1 or BRCA2 gene to those with verified HRD, and to those with proficient HRR. For example, in the germline BRCAmutated subpopulations, the improvement in median PFS with respect to the placebo arm ranged from 11.2 to 15.5 months in the various phase 3 trials $[4,5,7,8]$.

Even in patients treated with multiple lines, PARPi not given in a classical maintenance setting but as a continuous monotherapy have shown clinical benefit. In a multicenter, open-label, single-arm, phase 2 trial, niraparib was dosed daily as the fourth or later line of treatment and showed activity especially in patients with HRD-positive disease who responded to their last previous platinum-based therapy. In these patients, the clinical benefit rate (CBR; calculated as the proportion of patients experiencing any response or stable disease) for at least 16 weeks (CBR-16) was $74 \%$, whereas in HRD-negative platinum-refractory or platinum-resistant disease CBR-16 decreased to $18 \%$. Of special note, however, is the fact that in platinumrefractory/-resistant cancers exhibiting HRD including the BRCA mutations, the observed CBR-16 was still $33 \%$. When only BRCA-mutated cases of this subgroup were considered, the CBR-16 increased to as much as $43 \%$ [9].

Another chemotherapy-free, randomized phase 2 trial (AVANOVA-2) in platinum-sensitive recurrent disease compared niraparib monotherapy with the niraparib/bevacizumab combination. It is worth mentioning that $50 \%$ of the study patients had received two or more prior treatment lines. This proof of concept study clearly revealed that the combination of both drugs given as a definitive treatment for recurrent disease significantly improved PFS over niraparib alone. This improvement was regardless of HRD status or the chemotherapy-free interval, although the addition of bevacizumab appeared to be more beneficial in the subgroup with an interval of between 6 and 12 months as compared with patients with $>12$ months since the last chemotherapy. An additional exploratory analysis showed that the addition of bevacizumab to niraparib did not improve PFS significantly in BRCA-mutated cancers, whereas the combination was highly beneficial in BRCA-wildtype cases [10].

\section{PARPi in the first-line therapy of ovarian cancer}

Since the presentation of the SOLO-1 data at the 2018 European Society for Medical Oncology (ESMO) Meeting in Munich, Germany, olaparib was the first PARPi to move into first-line treatment for BRCAmutated high-grade serous and endometrioid ovarian cancers. This placebo-controlled randomized phase 3 trial, which only included germline or somatic BRCAmutated cancers, demonstrated an unprecedented improvement in PFS when olaparib was used as a maintenance treatment over 2 years after partial or complete response to first-line platinum-based therapy. At 3 years, $60.4 \%$ of the patients were progression-free in the experimental arm as compared to only $26.9 \%$ in the placebo arm. Furthermore, the median time to first subsequent therapy or death was the first secondary endpoint reached in that study and revealed a difference of more than 3 years in favour of the experimental arm. Moreover, it is worth noting that, after termination of the 2-year maintenance therapy, no approximation of both Kaplan Meier curves for PFS occurred, which strongly suggests that the beneficial effect of the olaparib maintenance therapy persists beyond treatment duration [11]. These results led the US Food and Drug Administration (FDA) and the European Medicines Agency (EMA) to approve olaparib for patients with BRCA-mutated HGOCs as a first-line maintenance treatment after a response to platinum-based chemotherapy.

Three further phase 3 placebo-controlled randomized trials regarding PARPi use in first-line treatment were presented at the 2019 ESMO Meeting in Barcelona. These clinical trials were performed with three different PARPi, namely veliparib, niraparib, and olaparib, but on substantially different patient populations, making a comparison of these drugs regarding their effectiveness absolutely inadmissible. Nonetheless, all three studies showed the unequivocal superiority of the PARPi treatment as compared with the placebo arm in the ITT population.

In the three-armed placebo-controlled VELIA Study, veliparib was administered concomitantly with the six cycles of platinum-based chemotherapy and as a 3year maintenance therapy [12]. The VELIA study was the only trial designed to enrol all patients with stage III-IV high-grade serous cancer regardless of BRCA status, surgical management, or response to platinum-based treatment. VELIA revealed that PARP inhibition with veliparib restricted to the duration of concomitant chemotherapy was not effective. How- 
ever, when veliparib was continued as maintenance therapy, PFS superiority was found for the ITT- (HR: 0.68) and the BRCA-mutated-population (HR: 0.44) when compared with the placebo arm. All this corroborated the significance of the maintenance approach of PARPi treatment in ovarian cancer. However, no benefit for veliparib over placebo was documented in the subcohort exhibiting HRD when BRCA-mutated disease was excluded as well as in HR-proficient cancers. Worthy of note is that in VELIA the HRD Score (Myriad myChoice HRD CDx ${ }^{\circledR}$ assay) threshold was set at 33 and was thus lower compared to the score cut-off of 42 used in all other studies [12].

The PRIMA study selectively included a population of very high-risk, advanced-stage patients, of which $67 \%$ were treated with neoadjuvant chemotherapy and interval debulking surgery. If primary surgery was performed, visible residual disease was a prerequisite. Furthermore, a $\geq 90 \%$ reduction in CA-125 from the original value at the end of chemotherapy was required, leading to a very high complete response rate $(75 \%)$ and a strongly preselected high platinumsensitive study population. The primary endpoints in the HRD and ITT population were reached for PFS with an HR of 0.43 and 0.62 , respectively, in favor of niraparip maintenance with respect to the placebo arm. Additional exploratory analyses also revealed a beneficial PFS for niraparib maintenance therapy in the subgroups of BRCA-mutated (HR: 0.40), HRD but BRCA-wildtype (HR: 0.50), and HR-proficient (HR: 0.68) disease. Regarding the HRR-proficient subgroup, calculations were based on an adjusted HR and relate to expected rather than actually observed values after adjustment for imbalances in key prognostic factors. Although statistically significant, the expected gain in median PFS is estimated at only around 2.4 months [13].

The platine, avastin, and olaparib in lst line (PAOLA-1) trial was the third maintenance PARPi study in HGOC first-line treatment where enrollment was regardless of BRCA status or surgical management [14]. The 2-year maintenance approach started after partial or complete response to platinum/taxane chemotherapy and consisted of bevacizumab with either olaparib or placebo. The addition of olaparib to bevacizumab maintenance therapy resulted in a significant improvement in the median PFS of 5.5 months in the ITT population (HR: 0.59), which was the primary endpoint of the study. Further exploratory subgroup analyses revealed a PFS improvement in BRCA-muted cancers of 15.5 months (HR: 0.31 ) and in HRD excluding BRCA-mutated cancers of 11.5 months (HR: 0.43). However, any benefit from the addition of olaparib to maintenance bevacizumab was documented in the HRR-proficient cancers (HR: 0.92). Taking the available data into consideration, the advantage of olaparib when added to bevacizumab maintenance therapy should be interpreted as an additive effect [14].
The most intriguing question when selecting patients that will probably not benefit from PARPi maintenance therapy is whether or not HRD testing should be performed and, if yes, how reliable commercially available tests are, especially with regard to the correct thresholds of the scores used in order to distinguish between HR deficiency and HR proficiency.

\section{Take home message}

In PARPi-naïve patients with recurrent HGOCs, PARPi should be generously used as maintenance therapy in BRCA-mutated cancers, as well as in other cancers showing unequivocal response to platinum-based chemotherapy regardless of the treatment line. In this context, it is worth highlighting that platinum sensitivity is still the best biomarker to predict response to PARPi treatment.

Moreover, in BRCA-mutated cancers, PARPi maintenance therapy (with or without bevacizumab) should be considered an integral part of front-line treatment. Therefore, BRCA testing should be offered to all ovarian cancer patients at primary diagnosis. BRCA-unrelated HRD cases in which olaparib and niraparib showed high effectiveness should also be treated with PARPi for front-line therapy. However, in this subgroup, testing of HRD status prior to therapy by reliable methods (either by commercially available specialized HRD tests or by academically established multigene analysis on new generation sequencing [NGS] platforms) should be vigorously recommended, especially in light of the concept of "precision medicine." This is especially true when response to platinum-based chemotherapy fails to be evaluated objectively due to complete tumor clearance during primary debulking surgery.

Nevertheless, the future use of PARPi in daily practice will be highly dependent on the terms that the FDA and EMA approve for the use of PARPi in the first-line treatment of ovarian cancer.

Funding Open access funding provided by University of Innsbruck and Medical University of Innsbruck.

Conflict of interest A.G. Zeimet, V. Wieser, K. Knoll, D. Reimer, and C. Marth declare that they have no competing interests.

Open Access This article is licensed under a Creative Commons Attribution 4.0 International License, which permits use, sharing, adaptation, distribution and reproduction in any medium or format, as long as you give appropriate credit to the original author(s) and the source, provide a link to the Creative Commons licence, and indicate if changes were made. The images or other third party material in this article are included in the article's Creative Commons licence, unless indicated otherwise in a credit line to the material. If material is not included in the article's Creative Commons licence and your intended use is not permitted by statutory regulation or exceeds the permitted use, you will need to obtain permission directly from the copyright holder. To view a copy of this licence, visit http://creativecommons.org/licenses/by/4.0/. 


\section{References}

1. Chambon P, Weill JD, Mandell P. Nicotinamide mononucleotide activation of new DNA-dependent polyadenylic acid synthesizing nuclear enzyme. Biochem Biophys Res Commun. 1963;11:39-43.

2. Helleday T, Petermann E, Lundin C, Hodgson B, Sharma RA. DNA repair pathways as targets for cancer therapy. Nat Rev Cancer. 2008;8(3):193-204.

3. Pothuri B. BRCA1- and BRCA2-related mutations: therapeutic implications in ovarian cancer. Ann Oncol. 2013;24(Suppl 8):viii22-viii7.

4. Pujade-Lauraine E, Ledermann JA, Selle F, et al. Olaparib tablets as maintenance therapy in patients with platinum-sensitive, relapsed ovarian cancer and a BRCA1/2 mutation (SOLO2/ENGOT-Ov21): a double-blind, randomised, placebo-controlled, phase 3 trial. Lancet Oncol. 2017;18(9):1274-84.

5. Pennington KP, Walsh T, Harrell MI, et al. Germline and somatic mutations in homologous recombination genes predict platinum response and survival in ovarian, fallopian tube, and peritoneal carcinomas. Clin Cancer Res. 2014;20(3):764-75.

6. Ledermann J, Harter P, Gourley C, et al. Olaparib maintenance therapy in platinum-sensitive relapsed ovarian cancer. NEngl J Med. 2012;366(15):1382-92.

7. Mirza MR, Monk BJ, Herrstedt J, et al. Niraparib maintenance therapy in platinum-sensitive, recurrent ovarian cancer. NEngl J Med. 2016;375(22):2154-64.

8. Coleman RL, Oza AM, Lorusso D, et al. Rucaparib maintenance treatment for recurrent ovarian carcinoma after response to platinum therapy (ARIEL3): a randomised, double-blind, placebo-controlled, phase 3 trial. Lancet. 2017;390(10106):1949-61.

9. Moore KN, SecordAA, Geller MA, etal. Niraparib monotherapy for late-line treatment of ovarian cancer (QUADRA): a multicentre, open-label, single-arm, phase 2 trial. Lancet Oncol. 2019;20(5):636-48.
10. Mirza MR, Åvall Lundqvist E, Birrer MJ, et al. Niraparib plus bevacizumab versus niraparib alone for platinum-sensitive recurrent ovarian cancer (NSGO-AVANOVA2/ENGOTov24): a randomised, phase 2, superiority trial. Lancet Oncol. 2019;20(10):1409-19.

11. Moore K, Colombo N, Scambia G, et al. Maintenance Olaparib in patients with newly diagnosed advanced ovarian cancer. NEngl J Med. 2018;379(26):2495-505.

12. Coleman RL, Fleming GF, Brady MF, et al. Veliparib with first-line chemotherapy and as maintenance therapy in ovarian cancer. N Engl J Med. 2019; https://doi.org/10. 1056/NEJMoa1909707.

13. González-Martín A, Pothuri B, Vergote I, et al. Niraparib in patients with newly diagnosed advanced ovarian cancer. N Engl J Med. 2019; https://doi.org/10.1056/ NEJMoa1910962.

14. Ray-Coquard I, Pautier P, Pignata S, et al. Phase III PAOLA-1/ENGOT-ov25: maintenance olaparib with bevacizumab in patients with newly diagnosed, advanced ovarian cancer treated with platinum-based chemotherapy and bevacizumab as standard of care. N Engl J Med. 2019;381(25):2416-28. https://doi.org/10.1056/ NEJMoa1911361

Publisher's Note Springer Nature remains neutral with regard to jurisdictional claims in published maps and institutional affiliations.

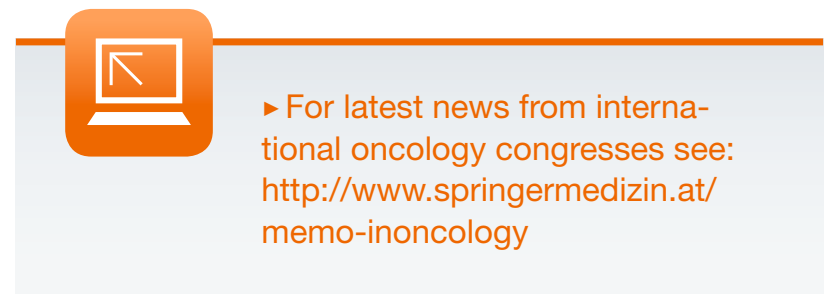

